\title{
CULTURAS JUVENILES Y CULTURA ESCOLAR ${ }^{145}$
}

\author{
Emilio Tenti Fanfani ${ }^{146}$
}

\section{Propósitos}

¿Qué es lo que se ofrece como educación escolar a los adolescentes y jóvenes de América Latina? ¿En qué medida lo que se ofrece responde a las condiciones de vida, necesidades y expectativas de las nuevas generaciones de latinoamericanos? Para responder a estas preguntas generales es preciso tener en cuenta los grandes cambios que caracterizan el desarrollo de la educación básica en nuestro continente. En un primer momento, dos fenómenos saltan a la vista: por un lado la masificación; por el otro, un cambio profundo en la morfología social de los nuevos inscriptos.

Los datos indican que la escuela para los adolescentes es una escuela en expansión. En muchos casos este crecimiento cuantitativo no fue acompañado por un aumento proporcional en los recursos públicos invertidos en el sector Demasiadas veces, "hubo que hacer más con menos". Es probable que la masificación estuviera acompañada de una disminución del gasto per cápita; así, se estiró al máximo el rendimiento de ciertas dimensiones básicas de la oferta, tales como recursos humanos, infraestructura física, equipamiento didáctico, etc. ${ }^{147}$

La escolarización, por una parte "crea juventud", es decir, contribuye fuertemente a la construcción de estos nuevos sujetos sociales. Pero por otra, la masificación produce una serie de transformaciones en las instituciones escolares. La vieja escuela media, reservada a las élites, hoy debe responder a la demanda de nuevos contingentes de ingresantes. Y la fuerza de la cantidad acarrea consecuencias de calidad. Las instituciones, en tanto que sistemas de reglas y recursos que estructuran las prácticas sociales y educativas, cambian de forma y significado. Los viejos dispositivos reguladores de la relación profesor-alumno, la relación con el conocimiento, garantizaban la autoridad pedagógica, y productores de un orden institucional, se erosionan cuando no saltan por los aires y dejan de ser eficientes y significativos en la vida de los actores implicados.

Pero la masificación está acompañada por un cambio muy significativo en la morfología social de los alumnos. No sólo los adolescentes y jóvenes que se escolarizan son más, sino que son diferentes. Por ejemplo, ingresan los que tradicionalmente estaban excluidos; a los "herederos y becarios" se agrega el grueso de la población, es decir, los hijos de los grupos sociales subordinados de las áreas urbanas, primero, y de las rurales, después. Estos recién llegados al nivel medio traen consigo todo lo que ellos son como

\footnotetext{
${ }^{145}$ Documento presentado al seminario "Escola Jovem: un novo olhar sobre o ensino médio", organizado por el Ministerio da Educaçao. Secretaria de Educaçao Média e Tecnológica. Coordenaçao-Geral de Ensino Médio (Brasilia, junio 7-9 de 2000).

${ }^{146}$ Sociólogo, consultor del IIPE-UNESCO en Buenos Aires. Profesor titular de sociología de la educación en la facultad de Ciencias Sociales de la universidad de Buenos Aires (Argentina).

${ }^{147}$ Durante el último quinquenio del siglo pasado, la matrícula en la enseñanza medía del Brasil creció el 57\%, "siendo el segmento de la enseñanza que ha crecido más en el periodo". Al mismo tiempo se señala este crecimiento fue desordenado y "sin las condiciones físicas adecuadas, sin espacios propios". Por ello según el Censo Escolar de 1998, el 55\% de los estudiantes cursan en el turno noche. (R. L. Berger Filho, 1999).
} 
clase y como cultura. De igual manera, los jóvenes y los adolescentes de hoy son distintos de los primeros "usuarios" de la educación media. Los grandes cambios en los modos de producción y en la estructura social y familiar, las transformaciones en el plano de las instancias de producción y difusión de significados (la cultura) afectan profundamente los procesos de construcción de las subjetividades. El poder del sistema educativo para formar personas, hoy es más relativo y relacional que nunca. Sus capacidades se miden en el sistema de relaciones que mantiene con la familia y las otras instancias que producen e imponen significaciones, en especial los medios masivos de comunicación y consumo cultural (J. C. Tedesco, 1995).

Todas estas transformaciones en la demografía, la morfología y la cultura de las nuevas generaciones pone en crisis la oferta tradicional de educación escolar. Los síntomas manifiestos y estridentes son la exclusión y el fracaso escolar, el malestar, el conflicto y el desorden, la violencia y las dificultades de la integración en las instituciones, y sobre todo la ausencia de sentido de la experiencia escolar para proporciones significativas de adolescentes y jóvenes latinoamericanos (en especial aquellos que provienen de los grupos sociales más excluidos y subordinados), con dificultades para ingresar, progresar y desarrollarse en instituciones que no han sido hechas para ellos. Todo parece indicar que aquellos que "llegan tarde" a la escuela (los adolescentes y jóvenes excluidos) ingresan a una institución ajena, y que por lo tanto no cumple ninguna función para sus proyectos vitales.

En las páginas que siguen me propongo exponer algunas reflexiones que espero ayuden a definir los principales problemas que surgen cuando se enfrentan dos culturas: la de los jóvenes y adolescentes y la que es propia de la tradición escolar. Creemos que comprender, interpretar, explicar y analizar es una dimensión necesaria de cualquier proceso encaminado a intervenir con alguna probabilidad de éxito en el campo de las políticas públicas. En especial, nos interesan aquellas orientadas a garantizar las mejores condiciones para facilitar el difícil proceso de construcción de la subjetividad y la inserción social de las nuevas generaciones de adolescentes y jóvenes latinoamericanos tanto al campo de la producción como al de la ciudadanía activa.

En síntesis, cuando los excluidos llegan a la educación media se produce el conflicto y el desencanto; conflicto, porque la universalización de la escolarización produce nuevos problemas y desafíos por las contradicciones entre características objetivas y subjetivas (expectativas, preferencias, actitudes, comportamientos, etc.) de la demanda y las características de la oferta (inadecuación institucional y empobrecimiento de la oferta escolar); y desencanto y frustración, porque cuando los pobres llegan a la escuela media se encuentran con que no existe ya correspondencia entre escolaridad, obtención del título del bachiller y determinadas sanciones materiales (puestos de trabajo e ingreso) y simbólicas (prestigio y reconocimiento social); porque llegan tarde, llegan en verdad a otro destino, y obtienen un objeto que tiene otro sentido y otro valor relacional, como es el caso de todos los objetos sociales.

\section{Sentido y valor de la enseñanza media en el mundo de hoy}

No es preciso abundar en argumentos para mostrar que la enseñanza media de hoy tiene un significado distinto al que tenía en el proyecto fundacional de los sistemas educativos occidentales. En un principio era una antesala de los estudios universitarios (y de ciertas posiciones en las burocracias públicas y privadas emergentes) y como tal estaba reservada sólo a los herederos, es decir, a los hijos de las clases dominantes y a 
algunos pobres meritorios (los "becarios"). Hoy la enseñanza media es más que eso. En verdad es otra cosa. Se trata de la última etapa de la escolaridad obligatoria. Vale no sólo como sendero que conduce a los estudios superiores (valor que conserva), sino que es algo así como el nuevo piso de la escolaridad obligatoria que en todas partes tiende a prolongarse hasta los 17 o 18 años de vida de los individuos. En las condiciones actuales, la llamada enseñanza media es una enseñanza "final" (un "techo") para la mayoría de la población, y un momento de un proceso de formación de una minoría, que tiende a prolongarse a lo largo de toda su trayectoria vital (educación permanente).

Esta nueva racionalidad cambia el sentido y afecta los viejos "modos de hacer las cosas" en las instituciones. En un principio, cuando se trataba de formar élites, la lógica de la selección impregnaba el quehacer de docentes y alumnos. La carrera escolar era como una carrera de obstáculos. Los "más capaces" de superarlos llegaban al final y adquirían el derecho de entrada a la universidad (en un principio no tenía sentido ninguna "prueba de ingreso" a la educación superior, la prueba era el título de bachiller). La práctica sistemática de los exámenes permitía distinguir a los exitosos de los fracasados. El fracaso era un fenómeno habitual y esperado en la experiencia escolar. Siempre los llamados eran más que los elegidos y todos los "jugadores" (maestros, familias, alumnos) conocían y compartían esta regla del juego y aceptaban sus desenlaces.

Cuando la enseñanza media se conviene en obligatoria, todos estos dispositivos dejan de tener sentido y, si persisten en su accionar, son una fuente de contradicción y conflicto. La reciente experiencia argentina de extensión de la obligatoriedad hasta el segundo año de la vieja secundaria (paso de la educación primaria obligatoria de 7 anos a la Educación General Básica de 9 años) está produciendo fuertes contradicciones entre los viejos mecanismos pedagógicos y disciplinarios, las expectativas de docentes y alumnos y las nuevas disposiciones legales y normativas ${ }^{148}$. Hoy la permanencia de los adolescentes en la escuela ya no es algo aleatorio o discrecional. Ni los alumnos ni los padres, ni los agentes escolares están en condiciones de determinar la inclusión o la exclusión escolar. Todos los adolescentes deben estar en la escuela.

Este es un mandato de la ley (la escolarización, al menos en el nivel básico fue siempre un derecho y una obligación) y, al mismo tiempo, un mandato social (del mercado de trabajo). Hoy la institución ha perdido la capacidad de imponer reglas que determinen la permanencia o el abandono escolar. En principio, todos deben ser contenidos. 4 Esta disposición determina la demanda y afecta fuertemente a la oferta. De esta forma, las familias y los jóvenes deben saber que ir o no ir a la escuela, al menos teóricamente, ya no es una cuestión de elección discrecional. $Y$ en cuanto a la instancia responsable de garantizar el cumplimiento de la obligatoriedad, es decir, al Estado, éste se ve obligado a ampliar la oferta escolar y a garantizar las condiciones mínimas de "educabilidad" de todos. De allí la tendencia a intervenir mediante programas de becas, subsidios y apoyo a los jóvenes y sus familias.

Pero el cambio de sentido y la obligatoriedad también determinan una serie de transformaciones en los dispositivos y procesos institucionales. El examen y la evaluación ya no pueden cumplir una función selectiva, sino estrictamente pedagógica, y los problemas de aprendizaje ya no se resuelven por la vía fácil y corta de la repetición y la exclusión; lo mismo puede decirse de los "problemas de conducta y disciplina". Sin embargo, las adaptaciones de las instituciones y las mentalidades no son simples efectos

\footnotetext{
${ }^{148}$ Incluso en el caso de la provincia de Buenos Aires donde vive un tercio de la población argentina, la obligatoriedad se prolonga hasta el final del viejo secundario, hoy denominado Nivel Polimodal (tres años luego de la EGB).
} 
automáticos de las transformaciones estructurales y legales. Por lo tanto, la contradicción tiende a transformarse en conflicto, y el desajuste entre las predisposiciones y los marcos normativos tiende a provocar el malestar. Por eso, la educación para los adolescentes y los jóvenes se convierte en el eslabón más crítico de las políticas educativas nacionales.

\section{Tres problemas en la escolarización masiva de los adolescentes y jóvenes}

Para ordenar la discusión propongo debatir estos nuevos desafíos de la escolarización generalizada de las nuevas generaciones alrededor de tres ejes problemáticos. El primero tiene que ver con el tema de la identidad y cultura de los adolescentes; el segundo, con el eje político de la modificación de los equilibrios de poder entre las generaciones; y el tercero, remite al tema del sentido de la experiencia escolar para los adolescentes y jóvenes. El hilo conductor que une estas tres líneas de reflexión tiene que ver, con el tema de la relación entre condiciones de vida y cultura de la población a escolarizar y la cultura propia de las instituciones escolares.

En este caso, hay un cuarto excluido y es precisamente el de la exclusión social que golpea fuertemente a muchos adolescentes y jóvenes latinoamericanos. La extrema desigualdad en la distribución de las oportunidades de vida hace que, para muchos de ellos, la escolarización, en sí misma, sea una experiencia literalmente imposible, algo que escapa completamente a su proyecto vital. En lo que sigue, esta determinación material no será tratada explícitamente, pero sí permanecerá como telón de fondo que determinará tanto las configuraciones culturales como los sentidos, las identidades y las trayectorias escolares de las nuevas generaciones de latinoamericanos ${ }^{149}$.

\section{Identidad y cultura de los adolescentes y jóvenes}

La adolescencia y la juventud son construcciones sociales. En otras palabras, son "clases de edad" que si bien tienen una base material biológica, sobre la misma se elaboran diversas representaciones relativamente arbitrarias e históricas. En realidad, lo que "existe" con una existencia casi igual a la de los objetos físicos es un continuo de edad. Es la sociedad la que produce determinados "cortes" y "rupturas" en el flujo del tiempo. Sabemos que existen niños y adolescentes, adolescentes y jóvenes, pero esas fronteras que marcan los límites no tienen una señalización material u objetiva. Los límites sociales son siempre "arbitrarios y conjeturales" (como decía J.L. Borges) y muchas veces imprecisos. Pero, en ciertos casos, es necesario reducir esa imprecisión fijando límites estrictos, homogéneos y fáciles de identificar. Este es el tipo de límite que se expresa en la Ley y en los dispositivos normativos. La "mayoría de edad", por ejemplo, está claramente establecida en los códigos y en las leyes de todas las sociedades. No son límites definitivos, pueden variar; pero sí son precisos. La incorporación al sistema educativo formal no es arbitraria; se ingresa a la escuela a una edad legal bien determinada.

Pero cuando se trata de la adolescencia y la juventud, sólo sabemos que existen, pero no estamos en condiciones de decir cuando empiezan y dónde terminan estas etapas de la vida ${ }^{150}$. No todos los que tienen la misma edad participan de la misma "clase de edad", ya que no todos los coetáneos comparten las mismas características y experiencias

\footnotetext{
${ }^{149}$ Detrás de las desigualdades y exclusión escolares están las desigualdades y exclusiones sociales. De allí la necesidad de imaginar y desarrollar estrategias de intervención integral donde el desarrollo de la infancia y la adolescencia ocupen un lugar central.

${ }^{150}$ Para una construcción teórica de las "clases de edad" ver M. Urresti, "Cambio de escenarios sociales: experiencia juvenil urbana y escuela, en E. Tenti Fanfani (comp.), Una escuela para los adolescentes, Losada, Buenos Aires, 2000.
} 
vitales (formar pareja, trabajar, alcanzar la autonomía económica, estudiar, etc.) ${ }^{151}$. Además, la propia experiencia escolar contribuyó a la creación de la juventud como una construcción social, es decir, como un tiempo de vida colocado entre la infancia y la condición de adulto, un tiempo de preparación y de espera. Por eso puede decirse que no siempre existió "juventud" y "adolescencia". La posición en la estructura de distribución de bienes materiales y simbólicos de la sociedad está determinando diversas formas de vivir la experiencia joven o adolescente, por lo tanto, no es un estado por el que necesariamente pasan todos los individuos en una sociedad determinada. En muchos casos hasta la propia experiencia de la infancia es un "privilegio" que se niega a muchos niños y niñas que viven en condiciones de pobreza extrema, tanto en el campo como en las grandes ciudades del continente.

Pero aquí, más que el debate teórico, por demás rico e interesante nos interesa saber cuáles son las características distintivas de los adolescentes y jóvenes respecto de los niños, en cuanto objeto de clasificación escolar. La vieja escuela primaria fue pensada y diseñada para los niños y la escuela media, pese a sus esfuerzos de adaptación, tiende a reproducir los mecanismos y estilos propios de la educación infantil. En otras palabras, en muchos casos, trata a los adolescentes como si fueran niños. Este es un factor que no pocas veces contribuye a explicar el malestar y el fracaso escolar en la enseñanza media.

Según Dubet y Martuccelli (1998), más allá de las significativas determinaciones de género, clase social, étnia, hábitat, etc., un estudiante del colegio secundado es diferente de un alumno de la escuela primaria ${ }^{152}$. Al menos pueden señalarse las siguientes particularidades observadas en Francia, pero que en cierta medida son válidas en el contexto escolar urbano de América Latina:

a. Diversidad de las "esferas de justicia ". Mientras el mundo de la infancia y la escuela está organizado alrededor de una gran "unidad normativa" que rige tanto en el ámbito escolar, como en la familia, el mundo del "colegial" está regido por la percepción de la existencia de diversos ámbitos de justicia; una regla se aplica en el recreo, otra entre los amigos, otra en el colegio, otra distinta en el ámbito familiar. Mientras que el niño mimado en la familia espera el mismo trato en la escuela, el adolescente percibe que existen distintos espacios de juego con distintas reglas. Por ejemplo, los, resultados escolares diferentes no deben engendrar tratamientos diferentes. Mientras que en la primaria, los que son buenos en conducta, también tienden a ser premiados en términos de notas y sanciones escolares, esta práctica se torna injusta en el colegio. En este ámbito, las clasificaciones escolares (calificaciones) tienden a diferenciarse de las calificaciones en el comportamiento (conducta).

b. Principio de reciprocidad. Mientras que, en la escuela, considera a la autoridad y al maestro como algo natural e indiscutido, el adolescente percibe que las instituciones (el colegio, pero también la familia) constituyen mundos complejos donde existen una diversidad de actores con intereses y "capacidades" diferentes. La "omnipotencia" del maestro tiende a ser sustituida por la visión más compleja y política de las relaciones y el juego (las alianzas, las estrategias, el uso del tiempo, etc.). El principio de reciprocidad quiere decir que la relación profesor-alumno no es unidireccional (el profesor tiene todo el poder y hace lo que quiere, mientras que el alumno sólo tiene que obedecer). El

\footnotetext{
${ }^{151}$ Uno podría preguntarse qué tienen en común las 400.000 niñas brasileñas que trabajan en el servicio doméstico con sus coetáneas de las clases medias altas y altas que estudian, disponen de tiempo libre, tienen determinados niveles y calidades de consumo cultural, etc.

${ }^{152}$ En la Argentina, se llama alumnos a los nulos que van a la escuela primaria. Los que frecuentan el colegio secundario y la universidad son estudiantes.
} 
adolescente tiende a pensar que el respeto, por ejemplo, debe ser una actitud recíproca y no sólo una obligación de él hacia sus profesores ${ }^{153}$.

c. La emergencia de estrategias escolares. El niño en la escuela percibe que sólo le basta ser aplicado y obedecer las reglas y a sus superiores (los padres y los maestros) para tener éxito en la escuela. En cambio, en el colegio los adolescentes perciben que "ser estudiante" es algo más complejo que seguir ciertos automatismos. Por el contrario, el adolescente percibe qué para tener éxito es preciso desplegar una estrategia, es decir, que se requiere hacer uso del cálculo, definir objetivos, elegir medios adecuados para los mismos, desplegar la acción en el eje del tiempo, saber esperar, etc.

d. Desarrollo de una subjetividad no escolar. Mientras los niños en las escuelas viven una "continuidad relativa", su estatuto de niño y su estatuto de alumno; los adolescentes en el colegio viven la experiencia de una tensión entre el estudiante y el adolescente. "Con la adolescencia —escriben Dubet y Martuccelli- se forma un 'sí mismo no escolar', una subjetividad y una vida colectiva independientes de la escuela, que 'afectan' a la vida escolar misma't Veremos más adelante que no todos los adolescentes logran articular en forma satisfactoria estés dos espacios de vida.

Más allá de estas particularidades genéricas, los adolescentes y jóvenes son portadores de una cultura social hecha de conocimientos, valores, actitudes, predisposiciones que no coinciden necesariamente con la cultura escolar, y en especial, con el programa que la institución se propone desarrollar.

Hubo un tiempo en que el mundo de la vida cotidiana se mantenía "afuera" y "alejado" de la cultura escolar. Los saberes legítimos, esos que la escuela pretende incorporar en los alumnos son saberes "consolidados" y en cierto modo "alejados" de la cotidianidad y la contemporaneidad. Esta distancia tenía una razón de ser en el momento constitutivo de la escuela y el estado modernos. La escuela tenía una misión civilizatoria, tenía una función de reeducación (como se decía en la época). En muchos casos, la distancia entre la cultura espontáneamente incorporada por los niños y la cultura que se quería inculcar era extrema. Por eso, la escuela tuvo una función misionera. La primera pedagogía era una tecnología de conversión, de allí la densidad, variedad e integralidad de sus tecnologías (al límite, el ideal era la pedagogía del internado).

Hoy resulta imposible separar el mundo de la vida del mundo de la escuela. Los adolescentes traen consigo su lenguaje y su cultura. La escuela ha perdido el monopolio de la inculcación de significaciones y éstas, a su vez, se inclinan a la diversificación y la fragmentación. Sin embargo, en demasiadas ocasiones, las instituciones escolares tienden al solipsismo y a negar la existencia de otros lenguajes y saberes y otros modos de apropiación distintos de aquellos consagrados en los programas y las disposiciones escolares.

Mientras que el programa escolar tiene todavía las huellas del momento fundacional (homogeneidad, sistematicidad, continuidad, coherencia, orden y secuencia únicos, etc.), las nuevas generaciones son portadoras de culturas diversas, fragmentadas, abiertas, flexibles, móviles, inestables, etc. La experiencia escolar se convierte a menudo en una frontera donde se encuentran y enfrentan diversos universos culturales.

Esta oposición estructural es fuente de conflicto y desorden, fenómenos que terminan, a veces, por neutralizar cualquier efecto de la institución escolar sobre la conformación de la

\footnotetext{
${ }^{153}$ Esta demanda se expresa claramente en esta declaración hecha por jóvenes alumnos de escuelas públicas de Río de Janeiro: "Se ele (el director.) nao me respeita, para que vou respeitá-lo? Eu respeito quem me respeita, quem me dá o respeito (...) Tem que haver a troca, se nao houver a troca quebra o elo da corrente, nao da" (Citado por M. C. de Souza Minayo y otros, 1999, p. 113).
} 
subjetividad de los adolescentes y los jóvenes (Jaim Etcheverry, 1999). Es preciso señalar que la contradicción y el conflicto entre cultura escolar y cultura social es más probable en el caso de los jóvenes de las clases sociales económica y culturalmente dominadas.

En estas condiciones, es posible que surjan tensiones entre la integración de los adolescentes a su "grupo de iguales" y su integración a las normas escolares. Cuando la distancia entre la cultura social incorporada por los muchachos y la cultura escolarcurricular es grande, el conflicto es un fenómeno factible en la experiencia escolar. Desde la clásica investigación de J. S. Coleman (1961), se conoce la oposición entre la subcultura adolescente y las normas escolares, que en muchos casos lleva a preferir la primera a la segunda. El conflicto y el predominio de la "atracción y el prestigio" en el grupo depares sobre el prestigio y los premios propios de la actividad escolar no es más que una de las situaciones presumibles. La armonización y "negociación" entre ambos universos culturales, dadas ciertas condiciones sociales e institucionales, es también un desenlace probable de esta tensión estructural. Las manifestaciones de este tipo de conflicto son bien conocidas y adquieren formas particulares en cada contexto nacional. En Francia, es común que los muchachos y las chicas del colegio tengan que optar entre dos figuras típicas: la del "bufón" y la del "payaso". Mientras que la primera figura representa el tipo ideal del alumno que Opta por cumplir con las reglas de la escuela, la segunda se aplica a quienes las desafían y prefieren ser los "primeros en el grupo" (los más valorados, reconocidos, populares, etc.) a costa de ser "los últimos" en la lista de méritos específicamente escolares (calificaciones, conducta, etc.). Los hijos de los grupos subordinados en muchos casos optan por esta estrategia, en la medida en que les resulta más difícil competir con éxito en la labor escolar.

El campo donde se juega la construcción de la subjetividad está dominado por tres actores básicos: la familia, los medios de producción y difusión de sentido, y las instituciones escolares. Pero la familia ha perdido fuerza y capacidad de estructurar las personalidades de las nuevas generaciones: la familia que la escuela todavía espera y quiere no es la de las nuevas generaciones. La incorporación de la mujer al mercado de trabajo, la modificación del equilibrio de poder entre los sexos y la división del trabajo en la familia, su desinstitucionalización y la cuestión social contemporánea, han modificado profundamente su papel como constructora de subjetividad.

No existe un curriculum social (es decir, familiar, mediático y escolar) único y coherente, y la escuela no tiene más remedio que prestar atención al hecho de que no posee una posición monopólica en este campo tan complejo (si es que alguna vez la tuvo). La simple toma de conciencia de esta complejidad contribuiría a redefinir y redimensionar, en forma crítica y creativa, el margen de maniobra y la eficacia propia de las instituciones escolares en la formación de las nuevas generaciones.

\section{Un nuevo equilibrio de poder entre las generaciones}

Otro factor que viene a poner en crisis los viejos dispositivos que organizaban la vida de las instituciones escolares para adolescentes y jóvenes se origina en los cambios en los equilibrios de poder entre los niños y jóvenes y los adultos. La edad siempre fue un principio estructurador de las relaciones de dominación en todas las sociedades y durante la segunda mitad del siglo XIX, cuando se sentaron las bases de las instituciones educativas capitalistas, la relación de poder entre las generaciones era mucho más asimétrica que en la actualidad. El mundo de los adultos (los padres, los maestros, los directivos, los "celadores", etc.) prácticamente monopolizaba el poder en las instituciones. Los alumnos tenían más deberes y responsabilidades que derechos y capacidades. Los reglamentos y dispositivos disciplinados de los establecimientos escolares constituyen 
una objetivación del poder omnipotente que tenían los adultos sobre las nuevas generaciones.

Por una serie de razones estructurales que se despliegan en el largo tiempo de la historia, el equilibrio de poder entre las generaciones ha sufrido cambios sustanciales (Elias, N., 1999). En la actualidad, aunque las relaciones intergeneracionales siguen siendo asimétricas y a favor de los "más grandes", esta asimetría se ha modificado profundamente en beneficio de las nuevas generaciones. Hoy los niños y adolescentes son considerados como sujetos de derecho. No sólo tienen deberes y responsabilidades como los mayores, sino que se les reconoce capacidades y derechos.

La Convención Internacional de los derechos del niño, incorporada prácticamente de forma universal (aun falta una firma significativa, la de los EEUU) es un indicador del grado de institucionalización alcanzado por estas nuevas relaciones de poder intergeneracional.

Las instituciones educativas tienen que tomar nota de esta realidad y transformar sus dispositivos, en especial aquellos que regulan las relaciones de autoridad entre profesores, directivos y alumnos, las que organizan el orden y la disciplina, y aquellas que estructuran los procesos de toma de decisión.

Habrá que reconocer que los adolescentes y jóvenes tienen derechos específicos (a la identidad, a expresar sus opiniones, a acceder a la información, a participar en la definición y aplicación de las reglas que organizan la convivencia, a participar en la toma de decisiones, etc.) y habrá que diseñar los mecanismos institucionales que garanticen su ejercicio (reglamentos, participación en cuerpos colegiados, recursos financieros, de tiempo y lugar, competencias, etc.) (Tenti Fanfani, 1999).

El reconocimiento de derechos a los adolescentes, aunado a la erosión de las instituciones escolares (producto de la masificación con subfinanciamiento y a la pérdida de monopolio en el campo de las agencias de imposición de significados), está en el origen de la crisis de la autoridad pedagógica como un efecto de institución. En las condiciones actuales, los agentes pedagógicos (maestros, directivos, expertos, etc.) no tienen garantizada la escucha, el respeto y el reconocimiento de los jóvenes. Pero la autoridad pedagógica, entendida como reconocimiento y legitimidad sigue siendo una condición estructural necesaria de la eficacia de toda acción pedagógica. El problema es que hoy el maestro tiene que construir su propia legitimidad entre los jóvenes y adolescentes. Para ello debe recurrir a otras técnicas y dispositivos de seducción. Trabajar con adolescentes requiere una nueva profesionalidad, la cual es preciso definir y construir.

\section{El problema del sentido de la escuela}

Los maestros no pueden dar por descontada su autoridad, sino que la tienen que construir en forma cotidiana. Y los alumnos deben darle un sentido a la experiencia escolar. En las condiciones del desarrollo actual éste no es automático, como en otras etapas del desarrollo escolar. Cuando los que frecuentan la enseñanza media son los "herederos y los becarios", es decir, los hijos de las clases dominantes y los pobres meritorios, tanto la autoridad pedagógica como el sentido de la escuela eran propios de la realidad. Hoy para qué ir a la escuela (a la escuela que tenemos, se entiende) es una pregunta pertinente, que la mayoría de los jóvenes y adolescentes se hacen a diario.

Es obvio que aquellos que no son capaces de dar una respuesta satisfactoria a esta pregunta tienen pocas razones para persistir en la carrera escolar. ¿Cuáles son las 
respuestas posibles y cuáles son las condiciones sociales que las determinan? Ellas son tres:

a) La obligación como sentido. Se va a la secundaria por una obligatoriedad social más que jurídica, "porque sí", porque no hay más remedio, porque es imposible no ir, y esto porque existe un sistema de contención familiar que, pese al vacío de la experiencia escolar y al malestar y padecimiento que puede llegar a producir, no asistir a la escuela no es una alternativa objetivamente factible para ciertos adolescentes y jóvenes. En este caso, el "ir al colegio" no es objeto de deliberación y elección, sino una experiencia ligada a una condición de edad.

b) La razón instrumental. Hay que estudiar por una razón puramente instrumental. Algunos adolescentes asumen la lógica de la postergación de beneficios presentes con el fin de beneficios mayores en el futuro. Si hoy me esfuerzo en los estudios (que en sí mismos no tienen mayor sentido), mañana seré alguien en la vida, podré ingresar a la universidad, podré lograr un buen empleo, recibiré mejor trato, etc.

c) El amor al conocimiento. Este emergente tiene que ver con la pasión, con la entrega incondicional al saber o a uno de sus campos. Esta inmersión en el juego, esta especie de illusio es una energía extremadamente poderosa que, según una representación pedagógica bastante difundida, se constituye en un recurso mayor para el éxito en la carrera escolar.

Estas tres disposiciones típicas ideales no están aleatoriamente distribuidas en la población, sino que son el producto de un conjunto de factores interrelacionados. Entre ellos cabe el mencionar los factores culturales y el lugar que ocupan los individuos en la estructura social. La relación gratuita, desinteresada y "pasional" con el conocimiento y la cultura, en general (el arte, las ciencias, etc.), es más probable que surja entre las clases más liberadas de las urgencias y presiones relacionadas con la sobrevivencia. Las clases medias, cuyo capital económico, social y cultural les permite aspirar al ascenso social, están objetivamente más predispuestas a desarrollar una lógica instrumental y a sacrificarse en el presente con el fin de lograr mejores recompensas en el futuro. Esta disposición al sacrificio y al esfuerzo sistemático es altamente valorada en el ámbito escolar ("persevera y triunfarás") y se asocia con los mejores rendimientos, tanto en términos de aprendizaje como de conducta escolar.

Los sectores sociales más excluidos de los valores sociales más preciados no están en condiciones objetivas de desarrollar una actitud estratégica entre las nuevas generaciones. Cuando objetivamente "no se tiene futuro", porque el mismo presente es incierto y se vive en situaciones límites, la simple idea de sacrificarse y esforzarse en función de recompensas diferidas en el futuro aparece como algo absurdo y literalmente impensable. Incluso la idea misma de futuro como tiempo "por venir" está ausente, cuando no se dan ciertas condiciones sociales básicas. Si no hay futuro, la lógica del cálculo y la relación entre medios y fines se agota en el tiempo corto del presente y la acción estratégica; en el sentido fuerte de-la expresión, no hay un horizonte donde explayarse. Demás está decir que lo anterior es una característica típica de muchos sectores sociales que viven situaciones extremas de exclusión social. Cuando este es el caso, las condiciones de "educabilidad" de los jóvenes se encuentran seriamente comprometidas.

\section{Características de una buena escuela para los jóvenes}

Dadas las condiciones en que se desarrolla la escolarización de los adolescentes y jóvenes latinoamericanos, es preciso preguntarse cuáles podrían ser las características que distinguen a una escuela adecuada a sus condiciones de vida, expectativas y derechos. 
Más que una respuesta, prefiero enunciar un listado de características que en principio aparecen como deseables y necesarias, sin por ello pretender agotar el tema, que por su complejidad requiere de análisis y reflexiones más especializadas. Pero a modo de propuesta aquí señalo las siguientes:

a) Una institución abierta que valoriza y tiene en cuenta los intereses, expectativas, conocimientos de los jóvenes.

b) Una escuela que favorece y da lugar al protagonismo de los jóvenes y donde los derechos de la adolescencia se expresan en instituciones y prácticas (de participación, expresión, comunicación, etc.) y no sólo se enuncian en los programas y contenidos escolares.

c) Una institución que no se limita a enseñar sino que se propone motivar, interesar, movilizar y desarrollar conocimientos significativos en la vida de las personas.

d) Una institución que se interesa por los adolescentes y los jóvenes como personas totales que se desempeñan en diversos campos sociales (la familia, el barrio, el deporte, etc.) y no sólo como los alumnos en tanto aprendices de determinadas disciplinas (la matemática, la lengua, la geografía, etc.).

e) Una institución flexible en tiempos, secuencias, metodologías, modelos de evaluación, sistemas de convivencia, etc., que toma en cuenta la diversidad de la condición adolescente y juvenil (de género, cultura, social, étnica, religiosa, territorial,etc.).

f) Una institución que forma personas y ciudadanos y no "expertos", es decir, que desarrolla competencias y conocimientos transdisciplinarios útiles para la vida, y no disciplinas y esquemas abstractos y conocimientos que sólo tienen valor en la escuela ${ }^{154}$.

g) Una institución que atiende a todas las dimensiones del desarrollo humano: física, afectiva y cognitiva. Una institución donde los jóvenes aprenden a aprender con felicidad y que integra el desarrollo de la sensibilidad, la ética, la identidad y el conocimiento técnicoracional.

h) Una institución que acompaña y facilita la construcción de un proyecto de vida para los jóvenes. Para ello deberá desplegarse una "pedagogía de la presencia" caracterizada por el compromiso, la apertura y la reciprocidad del mundo adulto para con los adolescentes y los jóvenes (A. C. Gómez Acosta, 2000 y 1997).

i) Una institución que desarrolla el sentido de pertenencia y con la que los jóvenes "se identifican".

\section{Cuatro peligros probables a controlar}

Pero las mejores intenciones pueden conducir a los peores resultados si no se tiene en cuenta que todo paradigma de intervención incuba efectos perversos, que es preciso conocer para controlar. Entre ellos, y para terminar, quiero mencionar los siguientes:

a. La condescendencia. Aconseja inventar escuelas para jóvenes pobres, contribuyendo así a la fragmentación social de la escuela y fortaleciendo la reproducción escolar de las desigualdades sociales y viceversa. Será preciso estar atento y controlar las buenas intenciones que invitan a concentrarse en los pobres y a ofrecer educación pobre para los jóvenes pobres.

b. El negativismo. Asociar la adolescencia y la juventud a situaciones indeseables, de peligrosidad social (delincuencia, enfermedad. drogadicción, embarazo adolescente, violencia, etc.), en síntesis, a la negatividad pura que sólo induce a la intervención preventiva. Para ello es preciso no olvidar que la adolescencia y la juventud son las edades de la energía, la fuerza, la belleza, la curiosidad, la imaginación, la creatividad, la

\footnotetext{
${ }^{154}$ En otro lugar hemos criticado la tendencia a la organización disciplinaria del curriculum de la educación básica y el predominio del teoricismo, el esquematismo y la abstracción sin sentido en la enseñanza escolar (E. Tenti Fanfani, "Pedagogía y cotidianidad", en E. Tenti Fanfani (2000).
} 
esperanza, el desinterés, la pasión. La sensibilidad, la entrega, la generosidad y otras riquezas asociadas.

c. El demagogismo juvenil y adolescente. Consiste en ofrecer comprensión, contención afectiva, respeto a la cultura joven, etc. sin desarrollar conocimientos y actitudes complejas y necesarias para la inserción social y política de los jóvenes en el mundo adulto. Algunas versiones del "populiculturismo" (es decir, la valoración voluntarista u oportunista de "las culturas de los jóvenes") acompaña y legitima muchas veces la exclusión respecto de los valores más valiosos y complejos de la cultura "adulta" y universal.

d. El facilismo. Consiste básicamente en una especie de reproducción escolar del modo de aprendizaje y de relación con la cultura que desarrollan los medios de comunicación de masa y de producción y circulación de culturas juveniles de masas (pedagogía del zapping, la espectacularización, el placer inmediato, el desprecio por la complejidad, el esfuerzo y el trabajo escolar, la disciplina, el carácter acumulativo del desarrollo de la cultura, etc.).

Pero no basta el ideal. Para cambiar el mundo de la escuela pública se requieren al menos tres ingredientes: a) hay que saber qué hacer y cómo hacerlo; b) hay que tener voluntad política y poder efectivo; c) hay que movilizar varios recursos significativos (al menos gente competente, tecnologías adecuadas, dinero y tiempo suficientes). Sin estos requisitos, las reformas se quedan en los papeles y nunca llegan a las aulas.

En síntesis, y para terminar, es probable que la escuela para los adolescentes sea una construcción, en la medida en que la propia adolescencia es una edad "nueva" y en plena transformación. Y como "todos los adolescentes no son iguales", habrá que pensar en formas institucionales lo suficientemente diversificadas y flexibles como para dar respuestas adecuadas a los múltiples condiciones de vida y expectativas de las nuevas generaciones. Quizás una de las claves del éxito sea comprender que una escuela para los adolescentes deberá ser, al mismo tiempo, una escuela de los adolescentes, es decir, una institución donde las nuevas generaciones no son simples poblaciones objetivo, sino protagonistas activos y "con derechos".

\section{Bibliografía}

Berger Filho, R. L. (1999). Enseñanza Media: Los desafíos de la inclusión. Documento presentado por el autor al Seminario "Cambios en la educación secundaria. Análisis de procesos europeos y latinoamericanos", organizado por el IIPE- UNESCO en Buenos Aires, noviembre de 1999.

Coleman, J.S. (1961). The adolescent society. The Free Press. Nueva York.

De Souza Minayo, M. C. E y otros (1999). Fala, galera. Juventude, violencia e cidadania no Rio de Janeiro. Editora Garamond/UNESCO, Río de Janeiro.

Dubet François y Martuccelli Danilo (1998). En la escuela. Sociología de la experiencia escolar Losada, Buenos Aires.

Elias N. (1999). La civilización de los padres. Editorial Norma. Bogotá.

Gómez da Costa, A. C. (2000). En E. Tenti Fanfani (2000), Una escuela para los adolescentes. Reflexiones y propuestas. Editorial Losada, Buenos Aires.

Jaime Echeverry, G. (1999). La tragedia Educativa. Fondo de Cultura Económica. Buenos Aires.

Tedesco, J. C. (1995). El nuevo pacto educativa Anaya, Madrid. 
Tenti Fanfani, E. (1999). Más allá de las amonestaciones. Cuadernos de la UNICEF. Buenos Aires.

Tenti Fanfani, E. (ed.), (2000). Una escuela para los adolescentes. Reflexiones y propuestas. Editorial Losada, Buenos Aires. 